\title{
Effect of acupuncture on menopausal vasomotor symptoms (VMS): A study protocol for a randomized controlled clinical trial
}

\section{Anna Bogachko}

Shanghai Municipal Hospital of Traditional Chinese Medicine

\section{Shanshan Li}

Shanghai Municipal Hospital of Traditional Chinese Medicine

Tian Li

Shanghai Municipal Hospital of Traditional Chinese Medicine

\section{Xuan Yin}

Shanghai Municipal Hospital of Traditional Chinese Medicine

\section{Ping Yin}

Shanghai Municipal Hospital of Traditional Chinese Medicine

\section{Lixing Lao}

University of Hong Kong

Shifen Xu ( $\boldsymbol{D}$ xu_doctor@hotmail.com )

Shanghai Municipal Hospital of Traditonal Chinese Medicine, Shanghai University of Traditional Chinese Medicine https://orcid.org/0000-0001-5595-3480

\section{Study protocol}

Keywords: Vasomotor symptoms (VMS), Hot flashes, Menopause, Acupuncture, Randomized controlled trial (RCT)

Posted Date: March 28th, 2019

DOI: https://doi.org/10.21203/rs.2.503/v1

License: (c) (i) This work is licensed under a Creative Commons Attribution 4.0 International License. Read Full License 


\section{Abstract}

Background During menopause, many women are troubled by vasomotor symptoms (VMS) when hot flashes, night sweats, or both, interfere with normal daily life. Both hormone therapy $(\mathrm{HT})$ and taking antidepressants are common ways to reduce VMS but they have associated health risks such as breast cancer and thromboembolic events. Acupuncture is found to be a safe treatment option to reduce VMS and recent studies have demonstrated positive results applying personalized selections of acupuncture points. Hence, we hypothesize acupuncture, according to Chinese Medicine's basic principle of pattern differentiation, can reduce both frequency and severity of VMS in menopausal women. We designed a randomized controlled trial to test the hypothesis using a wait-list group as a control group and pattern differentiation to find an optimal therapeutic treatment. Method/Design The study is a single-center, pragmatic, randomized control trial with two parallel arms, to assess the effectiveness of acupuncture in 60 women in the Shanghai international and Chinese community with peri- and postmenopausal VMS. Eligible participants will be randomly assigned into two groups on a ratio 1:1. An acupuncture group of 30 women will twice weekly receive totally 12 acupuncture treatments and we will follow their treatment response through their VMS self-rating journal entries recorded at baseline, during intervention and twice post intervention as primary outcome. A waitlisted control group will record the same self-ratings of their VMS for purposes of comparison. After the six-week wait period is over, they will receive the same 12 sessions acupuncture treatment. As a second outcome, menopause-specific quality of life indicators will be assessed at baseline and twice post intervention by all participants. Discussion If the outcome of this study confirms acupuncture to be an effective and safe management method to reduce VMS in menopausal women, we expect further investigations and trials to clinically find optimal acupuncture treating strategies.

\section{Background}

Menopause is a natural stage in a woman's life and marks the permanent completion of menstruation and of her ability to reproduce. During this transition, between the ages of $45-60$ years old, almost $80 \%$ of women in the western world are troubled by vasomotor symptoms (VMS) including hot flashes and night sweats[1-3]. For more than half of these women, unpleasant symptoms last seven to ten years and can severely affect sleep, comfort and quality of life[1, 4-6]. In the Chinese population, $15 \%$ of women report VMS symptoms[7].

Currently, the most common therapies for VMS are hormone therapy (HT) and taking antidepressants, but both carry risks and unwanted side-effects, such as risk of breast cancer, heart palpitations, dizziness and thromboembolic events that can cause women to seek alternative treatments[8,9]. Considering complementary and alternative medicine (CAM) such as black cohosh, phytoestrogen, yoga, acupuncture etc. has received a lot of attention as possible remedies to reduce VMS in menopausal women, but more studies about their effectiveness are needed[10]. 
Acupuncture is considered a safe treatment with few adverse events and has in previous studies been found to relieve VMS during peri- and post-menopause[8, 9, 11-13]. One explanation for acupuncture's efficacy in treating these symptoms is that the insertion of needles at certain acupuncture points causes the release of -endorphins in the hypothalamus. -endorphins inhibit the vasodilator calcitonin-gene related peptide (CGRP) in the thermoregulatory center which is increased in plasma during menopausal flashes. When CGRP in this way is reduced, vasomotor symptoms are reduced[8, 14]. Acupuncture can exert this kind of influence on the central nervous system and probably also affects serotonin and noradrenalin activity and thus has the potential to influence the thermoregulatory center in two ways, making it more stable[14].

Traditional Chinese Medicine (TCM) is built on syndrome differentiation, where patients are acknowledged to have different causes and patterns of a given disease or symptom. Therefore, according to TCM, women experiencing VMS should be treated with different acupuncture point selections $[8,11,15]$. A two-year expert panel consensus study agreed on syndrome patterns, symptoms and acupuncture points treating postmenopausal women with VMS [15]. Point selection in studies on VMS has varied, but TCM approaches using a larger selection counting for TCM syndromes have been most successful $[8,11,16]$. In addition, recent studies indicate that control measures other than sham needling can have reliable results treating VMS $[8,11,13,16]$. A novel study about VMS concluded it was beneficial using a wait-list control group both with regard to short term benefits as well as for long term results[8].

We designed this trial to explore the efficacy of a TCM approach to acupuncture treatment for VMS.

\section{Methods And Design}

\section{Hypothesis}

Based on studies of menopausal VMS, we suggest that a personalized standard combination of acupuncture points according to the basic treating principle of TCM will be beneficial to reduce VMS in peri- and postmenopausal women and further enhance their quality of life.

\section{Objectives}

1. To assess clinical evidence that demonstrates whether or not pragmatic personalized acupuncture is beneficial for menopausal VMS

2. To assess clinical evidence that demonstrates whether or not acupuncture enhances women's perceived health related quality of life during peri- and post-menopause

3. To find long term efficacy of acupuncture on VMS and health related quality of life

\section{Design}


The design is a single-center, pragmatic randomized controlled trial with two parallel arms, developed according to the Standard Protocol Items: Recommendation for Interventional Trials Statement (SPIRIT) [17]. We will follow the Standards for Reporting Interventions in Clinical Trials of Acupuncture (STRICTA) [18] throughout the trial. The procedure flow chart and details about data collection can be found in Figure 1.

\section{Methods}

Ethical approval (2018SHL-KY-10) has been obtained from the ethics committee of the Shanghai Municipal Hospital of Traditional Chinese Medicine affiliated with the Shanghai Traditional Chinese Medicine University in September 2018. A recruitment notice seeking women experiencing VMS, including hot flashes and night sweats, will be posted on billboards in the hospital. Because of the high incidence of VMS and patients' willing for complementary therapy in China, we're confident to recruit enough participants for this trial. A total of 60 women in peri- or post-menopause will be recruited and first screened via phone, social media or email. Those interested to participate in the study are asked to come to the hospital to receive a journal along with careful instructions how to self-rate their VMS including frequency and severity for seven consecutive days $[19,20]$.

\section{Inclusion criteria}

Eligible to participate in this two-year study are peri- and postmenopausal women between 45 and 60 years old, experiencing vasomotor symptoms more than four times per 24 hours during seven consecutive days of observation, according to VMS journal entries. Definition for experiencing perimenopause is self-defined amenorrhea during three or more months and definition for postmenopause is to have had amenorrhea for 12 or more months [8].

\section{Exclusion criteria}

Women with a history of cancer within the past five years, surgical menopause, heart valve disease, poorly controlled diabetes mellitus, organ transplant, mental disease, overt drug dependency or inability to complete study forms will not be included in the study. Additionally, women having initiated or changed their dose of antidepressant in the last three months or having initiated or changed their dose of any treatment for VMS in the last month will be excluded $[8,11]$.

\section{Procedure}

The women experiencing more than four VMS per 24 hours including both hot flashes and night sweats during seven consecutive days will be asked to come to the hospital for a second visit to do a baseline diagnosis according to TCM syndrome differentiation and for practitioners to take notes about current health situation and possible medication. All participants will be informed about the procedures of the study and sign a written consent. They will receive same advice on self-care and health preservation. Using the VMS self-rating journal completed by each participant we will receive a mean score for both frequency and severity to be regarded as baseline for their VMS and moreover, a first self-rating of 
perceived overall health situation will be completed through the Menopause-Specific Quality of Life questionnaire (MSQoL).

Next, randomization will be performed with a 1:1 allocation ratio. Women assigned to the acupuncture group $(n=30)$ will get 12 sessions of acupuncture treatment during a 6-week period while those assigned to a wait-list group $(n=30)$ will take same daily or weekly notes as required. First after having completed their six-week wait period, the control group will receive 12 sessions of acupuncture treatment.

Daily self-reports on VMS frequency and severity will be done using a small pocket size journal consisting of seven days' rating scales where ten scales per day (frequency) are marked 1-10 to represent severity of VMS. Participants will be asked to complete these self-reports in four rounds during the course of the study: (1) at the baseline assessment, (2) after the third week of intervention (mid-interval), (3) after the sixth week of intervention (post-treatment) and (4) at the 12-week interval after the last intervention to assess information on the long-term effects of acupuncture treatment. See Figure 1. The final self-report will indicate if long term reduction was sustained.

Both groups will rate their quality of life through the Menopause-Specific Quality of Life questionnaire (MSQoL) as a secondary outcome. This will be assessed three times: at the baseline assessment, posttreatment, and at the 12-week post-treatment.

\section{Intervention}

The intervention will consist of acupuncture and moxibustion. The acupuncture group will receive two sessions a week for six consecutive weeks. The wait-list group will receive 12 sessions for six consecutive weeks as well, after the completion of their six-week wait period. Follow up will be conducted 12 weeks after the last intervention.

Prior to any intervention, two acupuncturists from Shanghai University of Traditional Chinese Medicine, each with four years of experience in acupuncture practice, will jointly meet with each participant for optimal diagnosis. At this baseline diagnosis, each participant's individual TCM pattern related to VMS will be recorded.

Acupuncturists will ask questions about habits, current health situation, sleep patterns, do tongue diagnostics and pulse palpation, etc. according to TCM diagnostic practice. Therapeutic principles based on individual syndrome patterns will be found for each participant. Symptoms related to VMS such as depression, sleep problems, anxiety etc. will be considered during intervention. Needling points will be limited to the basic six points, with the option of four added points and moxibustion (see Table 1). Treatment protocol can be revised throughout the intervention period as the initial diagnosis based on TCM syndrome pattern in a patient might change one or several times.

For the interventions, three licensed acupuncture practitioners trained at Shanghai University of Traditional Chinese Medicine with three to five years acupuncture practice will be chosen. All 
acupuncturists are instructed through in-hospital meetings on how to proceed with the study format and interventions.

\section{Acupuncture prescription}

Through the application of TCM syndrome diagnostics the interventions will include and treat per participant, one or several of the following seven patterns: Kidney Yin deficiency, Kidney Yang deficiency, Yin and Yang deficiency, Heart and Kidney Yin deficiency, deficiency of Kidney and Spleen, Heart and Kidney not harmonized, Liver Qi stagnation and finally Kidney and Liver Yin deficiency with Liver Yang rising.

\section{Basic points}

Six points have been chosen as basic points for treating VMS $[11,15,21]$ : KI3, KI6, SP6, HT6, CV4 and BL23. Five of these points are needled at each intervention, alternating CV4 and BL23 every other session. These two points are on the stomach and back respectively and we will therefore alternate supine and prone position every other treatment session.

\section{Added points}

Participants will further receive needling based on their syndrome pattern. Hence, a maximum of four needling points may be added per session to regulate specific syndrome patterns. 'Added points' was created both to account for individual point selection and to have the flexibility to change points when patterns change during the six weeks of intervention. Additionally, limiting the number of points will, to some extent, standardize the acupuncture prescription.

Standard, sterile and disposable needles (0.25 x $40 \mathrm{~mm}$ - Jia Jian, China) will be inserted into the skin to a depth of 10-25 mm. Appropriate needle manipulation (including lifting, thrusting and rotation) will be applied for 10 seconds on each point so that the needling sensation (deqi sensation) can be accomplished at each point. Needles are retained for 30 minutes and moxibustion can be selected at one point per patient and session.

\section{Primary outcome measures}

The primary outcome will be change in mean daily frequency and severity of VMS according to journal entries made by participants before, during and after the interventions are completed. The control group will do the VMS recording with the same time interval for the purpose of comparison. A second posttreatment assessment will be carried out/performed 12 weeks after completed interventions (see Figure $2)[1,8,19,21]$. Self-rating of daily VMS in a journal has been reported a reliable and consistent measuring tool [20].

\section{Secondary outcome measures}


Secondary outcomes will be self-reported menopause-related quality of life, including the four domains somatic, psychological, vasomotor and sexual, measured through the Menopause-Specific Quality of Life questionnaire (MSQoL) at three assessments throughout the study (see Figure 2) $[6,8,22,23]$. This questionnaire was introduced in 1996 and thereafter used widely to measure women's self-estimated quality of life and life changes during menopause.

Participants will score 29 statements on a six-point Likert scale where the first rating is if present or not. If present, they will rate how bothersome on a zero (not bothersome) to six (extremely bothersome) scale. MSQoL was translated into Chinese including three translators or teachers of Chinese-English language all knowledgeable in TCM and medicine.

Adverse events such as fainting, dizziness or pain may occur during the treatment. Any adverse event (include any discomfort, symptoms, or diseases) should be reported by the patients and acupuncturists on time. All details of adverse events will be reported in the case report forms (CRFs). At the end of the study we will analyze these adverse events.

\section{Sample size calculation}

Sample size calculations were based on change in hot flash frequency score, referring to a previous trial on VMS [19]. At the end of the twelve-week intervention there was a $48 \%$ reduction for the acupuncture group (6.2 \pm 4.6$)$, a $28 \%$ reduction for the control group $(8.3 \pm 3.7)$ and $20 \%$ between-group difference $(P<0.001)$, with a pooled baseline of 12.5 flashes per $24 \mathrm{~h}$. Assuming this data can be applied to our trial, we used PASS15.0 with t-test for two independent samples to calculate our sample size. To detect a difference using a power of 90 , we need 7 participants in each group, using a significance level at $a=0.05$ and $\beta$-value of 0.1 . Due to our expectations of lower baseline scores and therefore harder to achieve clinically significant results in current trial we will add participants to allow us to detect a difference between the two groups. Moreover, assuming a $20 \%$ drop-out rate to consider we expand the sample size to 30 for each group, totally recruiting 60 participants.

\section{Randomization and blinding}

The participants will randomly be allocated to one of two trial groups: Acupuncture or Wait-list group, using a computer-generated randomization sequence with a block size of four, allocation ratio of 1:1. The assistant who is not involved with the data collection will allocate the participants into either acupuncture or wait-list group. She will pre-pack envelopes with the corresponding number according to the randomization schedule. Each patient will receive an envelope labeled with their name containing their numbered group assignment.

Since we set a wait-list group, the acupuncturists, the patients and the independent assistant who made the allocation won't be blinded to the group assignment. However, other researchers including the data analysts, outcome assessors, statistician will be blinded to group allocation.

\section{Data management and monitoring}


Patients' characteristics will be recorded in the CRFs, which will be kept in the researcher's office. Quality of CRF completion will be monitored during the trial. Two different researchers will enter the data twice for accuracy. Original data will be entered into the ResMan research Manager of the Clinical Trial Management Public Platform. Only the relevant users will have the account, and they will be trained and the system will be tested before it is officially launched to ensure that the system meets the trial requirements.

To improve the quality of our trial, the whole process will be conducted under the supervision of a qualified clinical trial expert (Lixing Lao). The Clinical Research Center of Drugs of the Shanghai University of Traditional Chinese Medicine will provide data monitoring with access to any interim results and will make the final decision to terminate the trial if necessary.

\section{Statistical Methods}

All data will be analyzed according to the Intention-to-Treat (ITT) principle, including data from any participants who have dropped out of the RCT during the trial. Statistical analysis will be performed using SPSS version 22. For the primary analysis comparing change in VMS between study groups, the analysis of covariance (ANCOVA) model will be used. Self-reported VMS data collected daily will be assigned a time variable used in the model, which is defined as number of weeks after randomization. In addition, MSQoL-scores in different dimensions will be calculated as mean scores based on the six-point Likert scale. The factor structure is evaluated through exploratory factor analysis using Principal Component Analysis (PCA) with Promax rotation[15].

\section{Discussion}

There have been a number of suggestions for diagnosing and treating VMS with acupuncture, including an international expert panel consensus [15], all being indicators for strategies in future research [8, 11, $15,21]$. Recent systematic trials have successfully used designs with a more open and flexible choice for point selection at treatment of VMS. This trial's presentation of eight syndrome patterns, and related choices of points is an attempt to use a TCM management that is somewhat standardized as a strategy. All interventions will be recorded with choices of points and moxa and data will be analyzed to evaluate the effects of acupuncture.

In addition, recent studies on VMS indicate that sham needling as a control measure provides poor results for acupuncture $[10,16,21,24]$. One possible explanation could be the choice of points at intervention in these studies, where the researchers mostly treated all participants with same standardized points based on the TCM diagnostic pattern kidney yin deficiency. The pattern of kidney yin deficiency was in another study [11] discovered to only count for $50 \%$ of the primary syndromes diagnosed by TCM in women with VMS. Moreover, sham-needling and its varied responses in a treatment setting has so far not been explained which might make it a less valid control measure [25-27]. Finally, interestingly, one analysis concluded there was no correlation between higher expectancy of the 
acupuncture treatment and the actual result [8]. To use a wait-list group as control group has had some interesting and favorable results $[1,8]$.

VMS in Chinese menopausal women are less common compared to western populations $[3,7]$. This study is designed to explore the efficacy of a TCM approach to acupuncture treatment for VMS but is also aiming at a better understanding about VMS in Chinese women.

\section{Abbreviations}

TCM: Traditional Chinese Medicine

VMS: Vasomotor Symptoms

HT: Hormone Therapy

RCT: Randomized Controlled Trial

CAM: Complementary and Alternative Medicine

MSQoL: Menopause-Specific Quality of Life questionnaire

CGRP: Calcitonin-Gene Related Peptide

SPIRIT: Standard Protocol Items: Recommendation for Interventional Trials Statement STRICTA: Standards for Reporting Interventions in Clinical Trials of Acupuncture CRF: Case Report Form

ITT: Intention-to-Treat

PCA: Principal Component Analysis

ANCOVA: Analysis of Covariance

\section{Declarations}

\section{Ethics approval and consent to participate}

This trial has been approved by the Ethics Committee of Shanghai Municipal Hospital of Traditional Chinese Medicine ((2018SHL-KY-10)). The purpose, procedures, and potential risks of the RCT will be explained to the participants. All participants shall give their written informed consent before joining the trial. 


\section{Trial status}

This manuscript is based on protocol version 1.0 (30th September, 2018). We began to recruit the participant in January, 2019 and the recruitment might be completed by December, 2019.

\section{Consent for publication}

Not applicable.

\section{Availability of data and material}

All data are fully available without restriction.

\section{Competing interests}

The authors declare that they have no competing interests.

\section{Funding}

The study is partly supported by grants from Shanghai Committee of Science and Technology, China (No 18401905200), Shanghai Hospital Development Center, China (No SHDC12016124), Shanghai Municipal Commission of Health and Family Planning, China (No 201640026) and State Administration of Traditional Chinese Medicine, China (Grant No. JDZX2015024). The content is solely the responsibility of the authors. The funding organizations had no role in the design and conduct of the study; collection, management, analysis, and interpretation of the data; preparation, review, or approval of the manuscript; and decision to submit the manuscript for publication.

\section{Authors' contributions}

SFX is the main researcher who provided conception and design of the study. $L X L$, head of the school of Chinese medicine in the University of Hong Kong, is the co-researcher, contributing to the design of the study and critical revision of the manuscript. AB provided good researcher direction and contributed to the writing of the draft. SSL and TL contributed to design of the protocol and the writing of the draft. XY and PY contributed to the statistical design, and are the project managers for the design of the randomization. All authors contributed substantially to the revision of this manuscript; and all authors read and approved the final manuscript.

\section{Acknowledgements}

Shifen Xu provided general support as the head of the Acupuncture Department in Shanghai Municipal Hospital of Traditional Chinese Medicine and was responsible for the design of this RCT and for communicating important protocol modifications to relevant parties. We would like to thank Shannon Ellis for her editorial support.

\section{References}


1. Avis NE, Crawford SL, Greendale G, Bromberger JT, Everson-Rose SA, Gold EB, Hess R, Joffe H, Kravitz $\mathrm{HM}$, Tepper PG et al: Duration of menopausal vasomotor symptoms over the menopause transition. JAMA Intern Med 2015, 175(4):531-539.

2. Kroenke CH, Caan BJ, Stefanick ML, Anderson G, Brzyski R, Johnson KC, LeBlanc E, Lee C, La Croix AZ, Park HL et al: Effects of a dietary intervention and weight change on vasomotor symptoms in the Women's Health Initiative. Menopause 2012, 19(9):980-988.

3. Avis NE, Ory M, Matthews KA, Schocken M, Bromberger J, Colvin A: Health-related quality of life in a multiethnic sample of middle-aged women: Study of Women's Health Across the Nation (SWAN). Med Care 2003, 41(11):1262-1276.

4. Freeman EW, Sammel MD, Sanders RJ: Risk of long-term hot flashes after natural menopause: evidence from the Penn Ovarian Aging Study cohort. Menopause 2014, 21(9):924-932.

5. Woods NF, Mitchell ES: Symptoms during the perimenopause: prevalence, severity, trajectory, and significance in women's lives. Am J Med 2005, 118 Suppl 12B:14-24.

6. Williams RE, Levine KB, Kalilani L, Lewis J, Clark RV: Menopause-specific questionnaire assessment in US population-based study shows negative impact on health-related quality of life. Maturitas 2009, 62(2):153-159.

7. Zheng J, Li J, Zhang L, Hu GH, Yu CQ, Zhang ZF, Ni S, Wei MJ: [Epidemiological investigation of perimenopausal women in Shanghai]. Zhong xi yi jie he xue bao $=$ Journal of Chinese integrative medicine 2009, 7(9):827-830.

8. Avis NE, Coeytaux RR, Isom S, Prevette K, Morgan T: Acupuncture in Menopause (AIM) study: a pragmatic, randomized controlled trial. Menopause 2016, 23(6):626-637.

9. Pirotta M, Ee C, Teede H, Chondros P, French S, Myers S, Xue C: Acupuncture for menopausal vasomotor symptoms: study protocol for a randomised controlled trial. Trials 2014, 15:224.

10. Kim DI, Jeong JC, Kim KH, Rho JJ, Choi MS, Yoon SH, Choi SM, Kang KW, Ahn HY, Lee MS: Acupuncture for hot flushes in perimenopausal and postmenopausal women: a randomised, shamcontrolled trial. Acupunct Med 2011, 29(4):249-256.

11. Borud EK, Alraek T, White A, Grimsgaard S: The acupuncture treatment for postmenopausal hot flushes (Acuflash) study: traditional Chinese medicine diagnoses and acupuncture points used, and their relation to the treatment response. Acupunct Med 2009, 27(3):101-108.

12. Sunay D, Ozdiken M, Arslan H, Seven A, Aral Y: The effect of acupuncture on postmenopausal symptoms and reproductive hormones: a sham controlled clinical trial. Acupunct Med 2011, 29(1):27-31. 
13. Kim KH, Kang KW, Kim DI, Kim HJ, Yoon HM, Lee JM, Jeong JC, Lee MS, Jung HJ, Choi SM: Effects of acupuncture on hot flashes in perimenopausal and postmenopausal women-a multicenter randomized clinical trial. Menopause 2010, 17(2):269-280.

14. Spetz Holm AC, Frisk J, Hammar ML: Acupuncture as treatment of hot flashes and the possible role of calcitonin gene-related Peptide. Evid Based Complement Alternat Med 2012, 2012:579321.

15. Alraek T, Borud E, White A: Selecting acupuncture treatment for hot flashes: a Delphi consensus compared with a clinical trial. J Altern Complement Med 2011, 17(1):33-38.

16. Dodin S, Blanchet C, Marc I, Ernst E, Wu T, Vaillancourt C, Paquette J, Maunsell E: Acupuncture for menopausal hot flushes. Cochrane Database Syst Rev 2013(7):CD007410.

17. Chan AW, Tetzlaff JM, Altman DG, Laupacis A, Gøtzsche PC, Krležajerić K, Hróbjartsson A, Mann H, Dickersin K, Berlin JA: SPIRIT 2013 Statement: Defining Standard Protocol Items for Clinical Trials. Annals of Internal Medicine 2013, 158(3):200-207.

18. Hugh MP, Altman DG, Richard H, Li Y, Wu T, Adrian W, David M: Revised STandards for Reporting Interventions in Clinical Trials of Acupuncture (STRICTA): Extending the CONSORT Statement. Journal of Integrative Medicine 2010, 8(9):35-46.

19. Borud EK, Alraek T, White A, Fonnebo V, Eggen AE, Hammar M, Astrand LL, Theodorsson E, Grimsgaard S: The Acupuncture on Hot Flushes Among Menopausal Women (ACUFLASH) study, a randomized controlled trial. Menopause 2009, 16(3):484-493.

20. Sloan JA, Loprinzi CL, Novotny PJ, Barton DL, Lavasseur BI, Windschitl H: Methodologic lessons learned from hot flash studies. J Clin Oncol 2001, 19(23):4280-4290.

21. Chiu HY, Pan CH, Shyu YK, Han BC, Tsai PS: Effects of acupuncture on menopause-related symptoms and quality of life in women in natural menopause: a meta-analysis of randomized controlled trials. Menopause 2015, 22(2):234-244.

22. Radtke JV, Terhorst L, Cohen SM: The Menopause-Specific Quality of Life Questionnaire: psychometric evaluation among breast cancer survivors. Menopause 2011, 18(3):289-295.

23. Painovich JM, Shufelt CL, Azziz R, Yang Y, Goodarzi MO, Braunstein GD, Karlan BY, Stewart PM, Merz $\mathrm{CN}$ : A pilot randomized, single-blind, placebo-controlled trial of traditional acupuncture for vasomotor symptoms and mechanistic pathways of menopause. Menopause 2012, 19(1):54-61.

24. Ee C, Xue C, Chondros P, Myers SP, French SD, Teede H, Pirotta M: Acupuncture for Menopausal Hot Flashes: A Randomized Trial. Ann Intern Med 2016, 164(3):146-154.

25. Organisation WH: Acupuncture: Review and Analysis of Reports on Controlled Clinical Trial. In.; 2002. 
26. Zotelli VL, Grillo CM, Sousa MD: A Case Report on the Effect of Sham Acupuncture. J Acupunct Meridian Stud 2016, 9(5):275-278.

27. Harris RE, Zubieta JK, Scott DJ, Napadow V, Gracely RH, Clauw DJ: Traditional Chinese acupuncture and placebo (sham) acupuncture are differentiated by their effects on mu-opioid receptors (MORs). Neuroimage 2009, 47(3):1077-1085.

\section{Table}

Table 1. Intervention and treatment details

\begin{tabular}{|c|c|c|}
\hline Intervention & Intervention details & Notes \\
\hline $\begin{array}{l}\text { Basic } \\
\text { acupoints }\end{array}$ & $\begin{array}{l}\mathrm{SP} 6, \mathrm{KI} 3, \mathrm{KI} 6, \mathrm{HT} 6, \mathrm{CV} 4 / \mathrm{UB} 23=5 \text { basic } \\
\text { acupoints per session }\end{array}$ & $\begin{array}{l}\text { Alternate supine and prone position } \\
\text { every other treatment }\end{array}$ \\
\hline $\begin{array}{l}\text { Added } \\
\text { acupoints }\end{array}$ & $\begin{array}{l}\text { Examples: KI7, KI10, LV3, LV8, GB20, ST36, } \\
\text { GV29, GV4, HT7, UB15 or others based on } \\
\text { patterns found }\end{array}$ & $\begin{array}{l}\text { Practitioner will be free to select a } \\
\text { maximum } 4 \text { of these or any } 4 \text { extra } \\
\text { points }\end{array}$ \\
\hline \multirow[t]{2}{*}{ Needle type } & \multirow{2}{*}{$\begin{array}{l}\text { Standard stainless-steel needles, sterile and } \\
\text { disposable }\end{array}$} & $0.25 \times 40 \mathrm{~mm}$ \\
\hline & & Jia Jian, China \\
\hline $\begin{array}{l}\text { Needle } \\
\text { sensation }\end{array}$ & With de-qi sensation & Depth of insertion: $10-25 \mathrm{~mm}$ \\
\hline Moxibustion & $\begin{array}{l}\text { Practitioner will be free to select one point for } \\
\text { moxibustion }\end{array}$ & Needle moxa or moxa box \\
\hline Duration & $30 \mathrm{~min}$ & \\
\hline
\end{tabular}

\section{Figures}




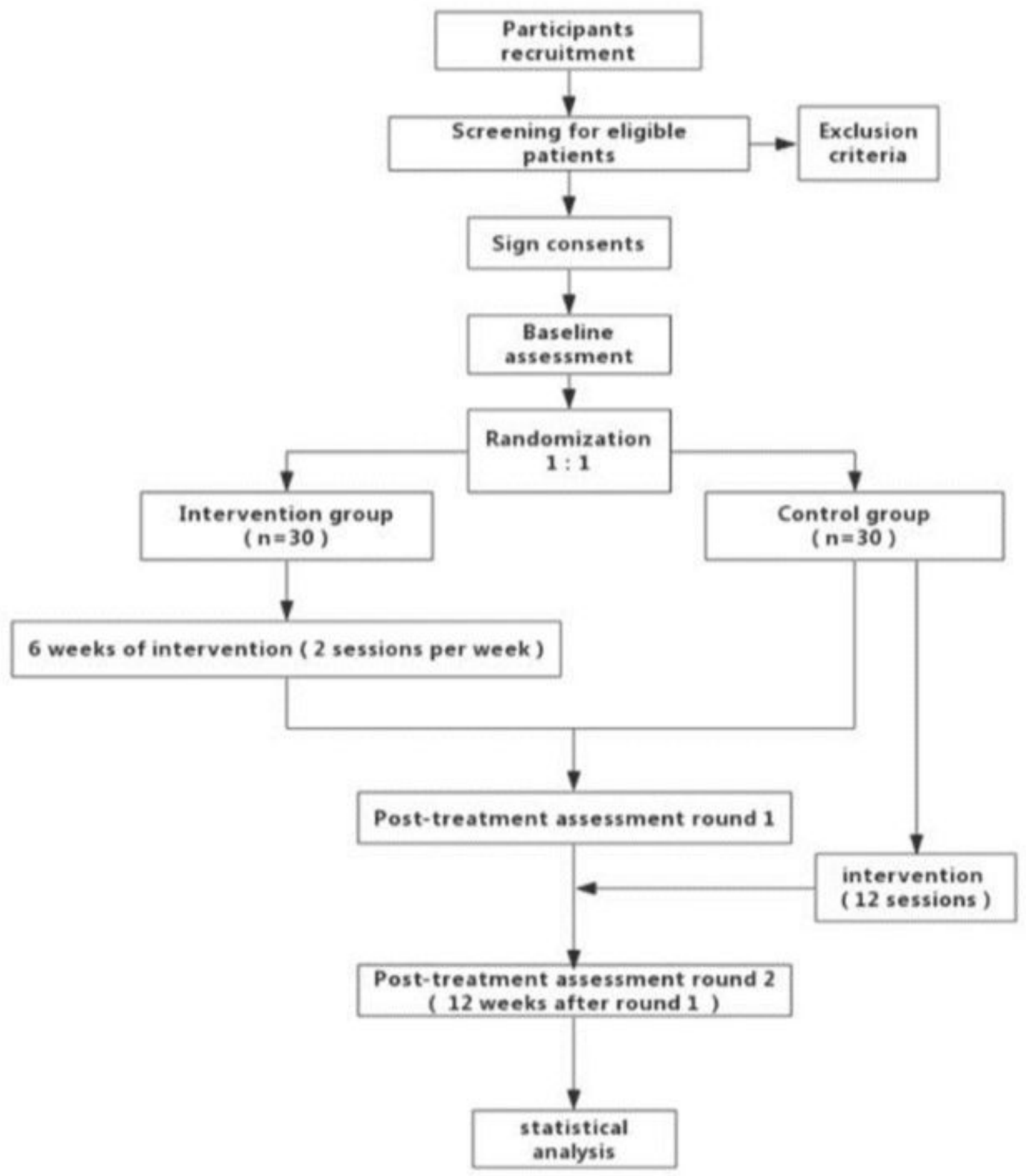

Figure 1

Study process. Figure 1 shows the design and procedure throughout the two years study. 


\begin{tabular}{|c|c|c|c|c|c|}
\hline & \multicolumn{5}{|c|}{ Study period } \\
\hline \multicolumn{1}{|c|}{ Timepoint } & Enrollment & Allocation & \multicolumn{2}{|c|}{ Intervention } & Post-intervention \\
\hline \multicolumn{1}{|c|}{ Enrollment } & & Week 0 & Week 4 & Week 7 & Week 19 \\
\hline Eligibility screen & & & & & \\
\hline Informed consent & $\mathrm{X}$ & & & & \\
\hline Medical history & $\mathrm{X}$ & & & & \\
\hline TCM diagnosis assessment & $\mathrm{X}$ & & & & \\
\hline Allocation & & $\mathrm{X}$ & & & \\
\hline Interventions & & & & & \\
\hline Acupuncture treatment & & & $\mathrm{X}$ & & \\
\hline Waiting-list & & & & $\mathrm{X}$ & \\
\hline Assessments & & & & & $\mathrm{X}$ \\
\hline Life questionnaire & & & $\mathrm{X}$ & $\mathrm{X}$ & $\mathrm{X}$ \\
\hline Adverse events & & & & $\mathrm{X}$ & \\
\hline
\end{tabular}

Figure 2

SPIRIT figure. Figure 2 shows the enrolment, interventions and data collection.

\section{Supplementary Files}

This is a list of supplementary files associated with this preprint. Click to download.

- SPIRITchecklist.doc 